\title{
Reproducibility of oxidative stress biomarkers in breath condensate: are they reliable?
}

\author{
I. Rahman
}

In this issue of the European Respiratory Journal, VAN HoYDONCK et al. [1] report a study about the reproducibility of oxidative stress biomarkers, such as hydrogen peroxide $\left(\mathrm{H}_{2} \mathrm{O}_{2}\right)$ and 8-isoprostane (a member of the $\mathrm{F}_{2}$-isoprostane class) in nonconcentrated exhaled breath condensate (EBC) obtained from healthy male smokers. The aim of their study was to identify whether these biomarkers could reproducibly be measured on different days of collection to enable identification of a susceptible population to lung diseases and monitoring of disease severity. The authors collected the EBC samples at three time points within 1 week (day 0 , day 3 or 4, day 7) from each healthy smoker. Their major findings were: 1) $\mathrm{H}_{2} \mathrm{O}_{2}$ and 8 -isoprostane were detected in only $47 \%$ and $36 \%$ of all EBC samples, respectively; 2) only three of 12 smokers had detectable 8-isoprostane concentrations on all three occasions (mean $4.6 \mathrm{pg} \cdot \mathrm{mL}^{-1}$; range $3.9-7.7 \mathrm{pg} \cdot \mathrm{mL}^{-1}$ ), whereas $\mathrm{H}_{2} \mathrm{O}_{2}$ was not detected on all three occasions in any of the smokers within 1 week; and 3) 8-isoprostane and $\mathrm{H}_{2} \mathrm{O}_{2}$ concentrations were below the limit of detection in many samples $\left(<3.9 \mathrm{pg} \cdot \mathrm{mL}^{-1}\right.$ for 8-isoprostane and $0.31 \mu \mathrm{M}$ for $\mathrm{H}_{2} \mathrm{O}_{2}$ ). They concluded that the levels of $\mathrm{H}_{2} \mathrm{O}_{2}$ and 8-isoprostane could not be reproducibly assessed in the EBC of healthy smokers because of their low concentration and/or the lack of sensitivity/specificity of the available assays. The other major factors that may affect the reproducibility would include intra- and inter-individual variability, sampling time and variability in dilution of respiratory droplets by water vapour [2]. The findings described in this study are not surprising, as many investigators have highlighted similar problems relating to the variation in reproducibility of these biomarkers in EBC [3, 4]. The variations in the reproducibility of these biomarkers could be due to methodological issues regarding sampling, storage and analytical techniques of EBC. Other confounding factors for the variation would include smoking, consumption of alcohol, caffeine and a diet rich in antioxidants.

The collection procedure, storage conditions and analysis of $\mathrm{H}_{2} \mathrm{O}_{2}$ and 8-isoprostanes in EBC are controversial [2]. In this study, VAN HAYDONCK et al. [1] have attempted to clarify the discrepancy associated with reproducibility of oxidative stress biomarkers in EBC obtained from healthy nonsmokers. By using a commercially available device (EcoScreen; Erich Jaeger GmbH, Hoechberg, Germany), they collected $\sim 1 \mathrm{~mL}$ of EBC from subjects breathing at a normal frequency, wearing a noseclip, for $15 \mathrm{~min}$. Previously investigators have used a variety of homemade condensing devices with different designs and found varying amounts (1-3 mL) of EBC after 15 min [2]. Now, with the use of EcoScreen, collection of EBC is being standardised in many leading laboratories, and this

Correspondence: I. Rahman, Edinburgh Lung Environment Group Initiative Laboratory, Medical Research Council Centre for Inflammation Research, The University of Edinburgh, Edinburgh, UK. Fax: 44 1316511558. E-mail: irfan.rahman@ed.ac.uk would no longer be a confounding factor contributing to the variations in biomarkers in EBC.

$\mathrm{F}_{2}$-isoprostanes are stable products of nonenzymatic lipid peroxidation of arachidonic acid that can be detected in all biological fluids. The potential disadvantage of measuring isoprostanes is that they are unstable at room temperature or on ice and rapidly degrade ex vivo. Agents, such as lipid peroxidation inhibitors and metal chelators, may be added prior to freezing to stabilise $\mathrm{F}_{2}$-isoprostanes. However, $\mathrm{H}_{2} \mathrm{O}_{2}$ evaporates readily due to its highly volatile nature. It is also known that the concentration of $\mathrm{H}_{2} \mathrm{O}_{2}$ decreases after a few days of storage at $-70^{\circ} \mathrm{C}$ and therefore EBC should be rapidly frozen at $-70^{\circ} \mathrm{C}$ or, preferably, assayed immediately [4]. The authors collected the EBC samples and divided them immediately into aliquots, stored at $-80^{\circ} \mathrm{C}$ and thawed at room temperature before analysis. Hence, the variability in concentration of these oxidative biomarkers may not be due to storage condition but possibly because they were not freshly analysed.

VAN HOYDONCK et al. [1], and many other investigators have measured $\mathrm{H}_{2} \mathrm{O}_{2}$ and 8 -isoprostane by $\mathrm{EBC}$ employing colorimetric and immunochemical methods, respectively [1, 4-6]. 8-Isoprostane was assayed by enzyme immunoassay kit and $\mathrm{H}_{2} \mathrm{O}_{2}$ by colorimetry using horseradish peroxidasecatalysed oxidation of tetramethylbenzidine as described by GALlati and PRACHT [7]. $\mathrm{H}_{2} \mathrm{O}_{2}$ can also be measured by fluorimetric techniques according to RUCH et al. [8] using the reaction substrates $\left(3,3^{\prime}, 5,5^{\prime}\right.$-tetramethylbenzidine, p-hydroxyphenylacetic acid, scopoletin, phenolsulfonphthalein, luminol 5-amino-1,2,3,4-tetrahydrophthalazine-1,4-dione, or homovanillic acid). These substrates can be added to samples immediately after collection and kept frozen until analysis.

By using these assays, the $\mathrm{H}_{2} \mathrm{O}_{2}$ levels in healthy young nonsmokers and smokers vary from 0.01 to $0.09 \mu \mathrm{M}$ and 0.10 to $0.75 \mu \mathrm{M}$, respectively [2-4]. Such variations $(60-80 \%)$ in $\mathrm{H}_{2} \mathrm{O}_{2}$ concentrations may be attributed to different storage conditions and/or analytical techniques used for EBC samples. The authors have also found that the limit of detection for $\mathrm{H}_{2} \mathrm{O}_{2}$ was $0.31 \mu \mathrm{M}$, which exceeded the other reported values $(0.1 \mu \mathrm{M})$. Although such variations have not been considered, variations due to the assay procedure employed may not be ruled out.

The majority of other investigators to date have used an immunoassay to measure 8-isoprostane concentrations in EBC. Using a commercial kit, VAN HoYDONCK et al. [1] have found the limit of detection of 8 -isoprostane to be $3.9 \mathrm{pg} \cdot \mathrm{mL}^{-1}$. Similarly, there are dozens of other studies that, using the same kit, reported a major variation in the levels of 8 -isoprostane in control healthy nonsmokers and healthy smokers $\left(6.2-22 \mathrm{pg} \cdot \mathrm{mL}^{-1}\right.$ and $6.7-29 \mathrm{pg} \cdot \mathrm{mL}^{-1}$, respectively), suggesting that the assay itself is variable. Hence, it may be argued the specificity of the commercial kit for this assay. For example, there may be cross-reactivity due to closely related 
species in biological fluids that could potentially interfere with the immunoassay. This emphasises the need for a highly specific assay system for measurement of 8-isoprostane. More specific and powerful analytical techniques, such as gas chromatography/negative ion chemical ionisation mass spectrometry (GC/MS) with an internal standard, could be considered for the assay. However, the levels of 8-isoprostane were only detected in three of 10 control subjects $(30 \%)$ in one study by GC/MS [9], suggesting that there is an immediate need to set a standard protocol for this technique, particularly in EBC. Additionally, other products of lipid peroxidation, such as malondialdehyde and 4-hydroxy-2-nonenal, can be derivitised immediately after sampling and be measured successfully by a more reliable high-performance liquid chromatography method $[10,11]$.

The level of oxidative biomarkers in EBC can be affected by smoking, diurnal variation and age. Cigarette smoking, in particular acute smoking, has been shown to cause increases of $\mathrm{H}_{2} \mathrm{O}_{2}$ and 8-isoprostane in EBC in healthy subjects [2, 6]. Also, smoking may give false values of oxidative stress biomarkers as cigarette smoke itself contains and/or forms $\mathrm{H}_{2} \mathrm{O}_{2}$ directly by chemical reactions through its interaction with the epithelial lining fluid. Hence, this aspect should be considered when interpreting the results obtained for markers of oxidative stress.

It is evident that the levels of 8-isoprostane and hydrogen peroxide cannot be reliably reproduced in exhaled breath condensate due to the reasons discussed above. In order to use these exhaled breath condensate markers for research/ clinical purposes, more sensitive and specific assays are required to reproducibly measure the biomarkers. Newer techniques, such as on-line measurement using sensitive biosensors, are being developed for more reproducible measurement of hydrogen peroxide. For example, it is possible to detect hydrogen peroxide on-line (real-time) using a silver electrode or by coating a platinum electrode or polymer with horseradish peroxidase [12, 13]. Similar enzyme detector systems may also be developed for real time monitoring of 8 -isoprostane. Thus, it is possible that oxidative stress biomarkers can be measured reproducibly provided they are measured immediately after sampling, on-line (real-time) or by more sensitive techniques, such as high-performance liquid chromatography and gas chromatography/negative ion chemical ionisation mass spectrometry. For that reason it is highly welcome and timely that the European Respiratory Society/American Thoracic Society Task Force "Exhaled Breath Condensate" is due to publish its methodological recommendations in the European Respiratory Journal within a couple of months.

\section{References}

1. Van Hoydonck PGA, Wuyts WA, Vanaudenaerde BM, Shouten EG, Dupont LJ, Temme EHM. Quantitative analysis of 8-isoprostane and hydrogen peroxide in exhaled breath condensate. Eur Respir J 2004; 23: 189-192.

2. Rahman I, Kelly F. Biomarkers in breath condensate: A promising new noninvasive technique in free radical research. Free Radic Res 2003; 37: 1253-1266.

3. Nowak D, Kalucka S, Bialasiewicz P, Krol M. Exhalation of $\mathrm{H}_{2} \mathrm{O}_{2}$ and thiobarbituric acid reactive substances (TBARS) by healthy subjects. Free Radic Biol Med 2001; 30: $178-186$.

4. van Beurden WJ, Harff GA, Dekhuijzen PN, van den Bosch MJ, Creemers JP, Smeenk FW. An efficient and reproducible method for measuring hydrogen peroxide in exhaled breath condensate. Respir Med 2002; 96: 197-203.

5. Dekhuijzen PN, Aben KK, Dekker I, et al. Increased exhalation of hydrogen peroxide in patients with stable and unstable chronic obstructive pulmonary disease. Am J Respir Crit Care Med 1996; 154: 813-816.

6. Montuschi P, Collins JV, Ciabattoni G, et al. Exhaled 8-isoprostane as an in vivo biomarker of lung oxidative stress in patients with COPD and healthy smokers. Am J Respir Crit Care Med 2000; 162: 1175-1177.

7. Gallati H, Pracht J. Horseradish peroxidase: kinetic studies and optimisation of peroxidase activity determination using the substrtes $\mathrm{H}_{2} \mathrm{O}_{2}$ and 3, 3',5,5' -tetramethylbenzidine. J Clin Chem Clin Bioch 1985; 23: 453-460.

8. Ruch W, Cooper $\mathrm{PH}$, Baggiolini M. Assay of $\mathrm{H}_{2} \mathrm{O}_{2}$ production by macrophages and neutrophils with homovanillic acid and horse-radish peroxidase. J Immunol Methods 1983; 63: 347-357.

9. Carpenter C, Price P, Christmas B. Exhaled breath condensate isoprostanes are elevated in patients with acute lung injury and ARDS. Chest 1998; 114: 1653-1659.

10. Larstad M, Ljungkvist G, Olin AC, Toren K. Determination of malondialdehyde in breath condensate by high-performance liquid chromatography with fluorescence detection. $J$ Chromatogr B Biomed Sci Appl 2002; 766: 107-114.

11. Corradi M, Rubinstein I, Andreoli R, et al. Aldehydes in exhaled breath condensate of patients with chronic obstructive pulmonary disease. Am J Respir Crit Care Med 2003; 167: $1380-1386$

12. Razola SS, Ruiz BL, Diez NM, Mark HB, Kauffmann JM. Hydrogen peroxide sensitive amperometric biosensor based on horseradish peroxidase entrapped in a polypyrrole electrode. Biosens Bioelectron 2002; 17: 921-928.

13. Thanachasai S, Rokutanzono S, Yoshida S, Watanabe T. Novel hydrogen peroxide sensors based on peroxidase-carrying poly[pyrrole-co-[4-(3-pyrrolyl)butanesulfonate] copolymer films. Anal Sci 2002; 18: 773-777. 